\title{
Multicast Resource Allocation with Opportunistic Scheduling in LTE Networks
}

\author{
Mohammed Algharem ${ }^{1}$, Mohammed Hasbullah Omar ${ }^{1}$, Ismat Aldmour ${ }^{2}$, Rahmat Budiarto ${ }^{2}$ \\ ${ }^{1}$ InterNetWorks Research Group, School of Computing, UUM-CAS, Universiti Utara Malaysia, \\ Sintok, Kedah, Malaysia \\ ${ }^{2}$ College of Computer Science \& Information Technology, Albaha University, \\ PO Box 1988, Albaha, Saudi Arabia \\ algharem.mhomar@internetworks.my
}

\begin{abstract}
Long Term Evolution (LTE) and beyond networks offer high bitrates with low latencies which makes them the best choice to handle the growing demand on multicast mobile services such as video streaming, and news broadcasting. In Conventional Multicast Scheme (CMS), the gain of multicast is exploited by transmitting data to all users simultaneously using the rate of Worst Channel Gain (WCG), which wastes network resources. In this paper, to better balance the tradeoff between the multicast gain and multiuser diversity, we propose a mechanism to select a threshold to split users into two subgroups and exploits the Opportunistic Multicast Scheme (OMS) to transmit the data. Simulation results show that the proposed mechanism improves the multicast performance in terms of increased throughput, decreased delay and Packet Loss Ratio (PLR) compared to existing mechanisms from the literature.
\end{abstract}

Index Terms-E-MBMS; LTE; Modulation and coding scheme; Opportunistic multicast scheme.

\section{INTRODUCTION}

Long Term Evolution (LTE) network was introduced by the Third-Generation Partnership Project (3GPP) and considered as the latest step towards the $4^{\text {th }}$ generation of radio technologies [1]. LTE offers a high throughput with low latency which makes it the best choice for Multimedia Service. LTE network exploits the benefits of Orthogonal Frequency Division Multiple Access (OFDMA), in which the transmitted data is multiplexed in frequency and time domains [1]. To face the rapid growth of demand on multicast services such as Internet video streaming, video conferencing, news broadcast, and weather forecasts, Evolved Multimedia Broadcast Multicast Service (EMBMS) as part of the $3 \mathrm{GPP}$ LTE standards was proposed to deal with multicast services [2]. Thus, E-MBMS is considered as the best choice when a number of subscribers request the same data at the same time [3], [4].

In unicast mode, each user sends its Channel Quality Indicator (CQI) feed- back to its base station which is called Evolved NodeB (eNodeB), in turn the eNodeB dynamically adjusts for that user the modulation type and code rate; combined together called the modulation and coding scheme (MCS). This improves the network resource utilization and increase the spectral efficiency (SE). On the other hand, E-

Manuscript received 4 November, 2017; accepted 2 June, 2018.
MBMS users listen to the same channel with different channel conditions. Therefore, the selected MCS level should satisfy all users and if the MCS level was selected according to the users who are suffering bad channel conditions (such as using worst channel gain (WCG), the channel will be more robust against error and failure, but, users with good channel conditions will sacrifice their opportunity to receive the data with high bit rates which, in turn, reduces the radio spectrum efficiency of such Conventional Multicast Schemes (CMS) [5], [6]. In other words, transmitting the data to a multicast group (MG) using the rate of WCG will increase the fairness between users at the expense of system throughput. Therefore, the performance of the multicast network is bounded by the user in worst channel condition who acts as a bottleneck phenomenon [7]. In [8], Zhang et al. proposed a mechanism to overcome the worst user bottleneck by using a Signal Interference and Noise Ratio (SINR) threshold to sacrifice the users who are expensive to cover. Users with G-factor less than the threshold will be excluded from link adaptation and packet scheduling. The G-factor (called static SINR) is obtained by considering shadowing and large-scale attenuation without fast fading. In fact, there are several mechanisms which have been proposed such as Average, Median, and maximum throughput (MT). However, by using these mechanisms, some users may fail to decode the transmitted data. In order to solve this problem, an opportunistic multicast schema (OMS) was proposed [9]. In OMS, the Median rate is used to serve the portion of users with rate equal or greater that the Median rate. In the second Transmission Time Interval (TTI), the eNodeB will retransmit the same data to remaining users who could not receive the data in the first transmission using their Median rate. The eNodeB will continue retransmission of the same data until all users receive the data. Indeed, OMS was proposed to utilize the multiuser diversity while maintaining the multicast gain as much as possible. Thus, the portion of users should be selected carefully. Let $\alpha$ denotes the portion of users that can be served in each Transmission Time Interval (TTI). Thus in the Median mechanism, $\alpha=0.5$.

In fact, splitting the multicast users to many subsets (or portions) will increase the multiuser diversity while degrading the multicast gain and vice versa. However, selecting the same number of users in each subset will not 
be the best configuration in all TTIs. To overcome this, in [10], [11], a dynamic OMS (D-OMS) with different portions (dynamic $\alpha$ ) was proposed. In each TTI, the D-OMS mechanism selects a portion of users that can increase the multicast throughput compared to the Median mechanism. However, the authors did not evaluate the D-OMS in term of delay. It is obvious that more subsets will increase the delay, especially when there is a scarcity in the available bandwidth.

Obviously, obtaining the best portions configuration, which leads to maximizing the multicast group throughput, is an NP-Hard problem. Moreover, the problem complexity increases exponentially as the number of available Radio Bearers (RBs) and groups of user increases. Thus, full research algorithm can be used to find the optimal configuration between all possible solutions, but it is not suitable to be used in real systems.

However, in multicast mode, due to the heterogeneity of users, allocating radio resources and adapting transmission rate will be the most challenging issues. The MCS level selected will directly impact the throughput and the SE in a multicast transmission mode [12], [13]. After all, no single MCS level can satisfy all users.

Moreover, many works on EMBMS over LTE are recently being carried out such as works in [14]-[19] to show state of the art of researches in the multicast over LTE networks.

In this work, a novel MCS selection mechanism to select a threshold that can be used with the OMS strategy to enhance the multicast performance is proposed. The proposed mechanism extends the work in [20], which takes the users dispersion into account by using the Standard Deviation $(S t D)$ of users' MCS to obtain a threshold. Thus, the selected threshold will be used to split users into two subsets, users with high channel gain (users whose MCSs are larger than or equal to the threshold) will form the first subset, whereas users with the worst channel gain (users whose MCS are less than the threshold) will form the other subset. The performance of the proposed mechanism is compared with other existing mechanisms without or with the added opportunity for repeated multicast retransmissions to their respective abnormal groups.

The rest of this paper is organized as follows: Section II, provides a brief introduction of AMC in E-MBMS. A background about well-known mechanisms is presented in Section III. Then, the proposed mechanisms for selecting the MCS are presented in Section IV. Simulation results are in Section V. Finally, conclusions and future work are presented in Section VI.

\section{ADAPTIVE MODULATION AND CODING}

Adaptive Modulation and Coding (AMC) is a key technique to adapt the data rates over the connection links in LTE networks in order to maximize system throughput and $\mathrm{SE}$ [21]-[24]. In AMC, the user's channel condition is reported back to the eNodeB packet scheduler which in turn chooses the most proper MSC level to this user. Each user measures the SINR for its channel, maps it to a corresponding CQI value, and sends it, periodic or aperiodic, to its eNodeB. The eNodeB will in turn use the CQI report to adjust the modulation type and code rate
(MSC level) for that user. The modulation types used in the transmissions can range from the more robust QPSK, to 16QAM, and to the highest throughput 64QAM. Each modulation type can also be coded at different rates. LTE define 15 MCS levels (represented in even numbers 0, 2, 4 ... 28 in Table I) each corresponds to one CQI level. A user close to the eNodeB who is in favourable channel conditions will report high CQI level (equal or close to 15) which entitles him to receive the data at a corresponding high MCS level (using a high rate 64QAM). On the other end, a cell edge user encountering worst channel conditions will report a low CQI (close to 1) which entitles him to receive the data using a corresponding low MCS level of low rate, but more robust, QPSK. Hence, link quality is preserved without the need for more power This situation in the cell is depicted in Fig. 1 [25], [26].

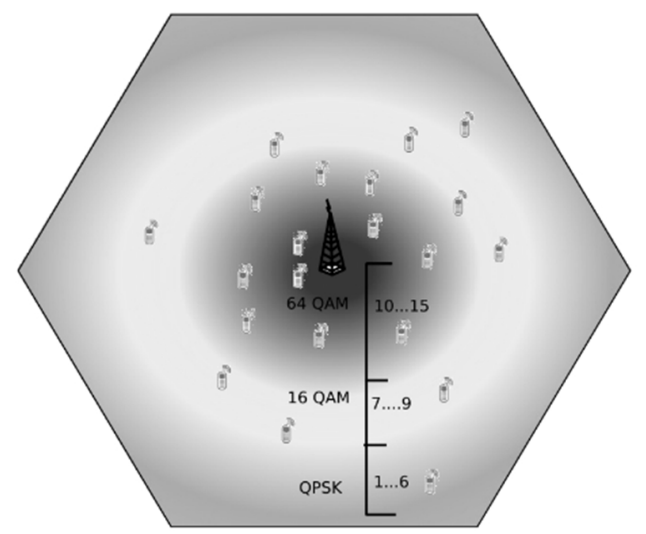

Fig. 1. Typical distribution of different modulations in the cell.

TABLE I. MAPPING OF CQI INDICES TO MCS LEVELS.

\begin{tabular}{|c|c|c|c|c|}
\hline CQI & MCS & $\begin{array}{c}\text { Type of } \\
\text { Modulation }\end{array}$ & $\begin{array}{c}\text { Code Rate } \\
\mathbf{x 1 ~ K b i t s / s e c ~}\end{array}$ & $\begin{array}{c}\text { SE } \\
\text { Bits/Hz }\end{array}$ \\
\hline 1 & 0 & QPSK & 78 & 0.1523 \\
\hline 2 & 2 & QPSK & 120 & 0.2344 \\
\hline 3 & 4 & QPSK & 193 & 0.3770 \\
\hline 4 & 6 & QPSK & 308 & 0.6016 \\
\hline 5 & 8 & QPSK & 449 & 0.8770 \\
\hline 6 & 10 & QPSK & 602 & 1.1758 \\
\hline 7 & 12 & 16QAM & 378 & 1.4766 \\
\hline 8 & 14 & 16QAM & 490 & 1.9141 \\
\hline 9 & 16 & 16QAM & 616 & 2.4063 \\
\hline 10 & 18 & 64QAM & 466 & 2.7305 \\
\hline 11 & 20 & 64QAM & 567 & 3.3223 \\
\hline 12 & 22 & 64QAM & 666 & 3.9023 \\
\hline 13 & 24 & 64QAM & 772 & 4.5234 \\
\hline 14 & 26 & 64QAM & 873 & 5.1152 \\
\hline 15 & 28 & 64QAM & 948 & 5.5547 \\
\hline
\end{tabular}

In Point to Point (PtP) transmission mode, AMC can be easily and efficiently implemented as the proper MCS level for any user can be selected based on user's CQI feedback report. On the contrary, in Point to Multi-Point (PtM) mode, such as multicast services, a group of users, suffering different channel conditions, listen to the same multicast channel. This entitles that eNodeB should adopt a proper MCS level which suits all of them in terms of throughput and robustness [27], [28]. The selected MCS level will directly impact the multicast throughput which makes this selection very important to improve SE in OFDMA-based multicast systems [29]. 


\section{The Well KNOWn Multicast MCS SELECTION MECHANISMS}

\section{A. Worst Channel Gain Condition}

The easiest way to select an MCS level in PtM is to select the MCS level according to the worst case user among EMBMS users group. In the Worst Channel Gain mechanism (WCG), the lowest MCS of all users is selected. This mechanism causes a degradation in the system performance in term of SE and throughput since the WCG does not take into account the users' distribution. The users who are in good channel conditions sacrifice their opportunity to receive the data with high bit rate (using high MCS level). On the other hand, this mechanism provides the E-MBMS service with high robustness, suppresses errors, and ensures fairness, on the account of the SE [27]-[31].

In order to understand the effectiveness of the selected MCS level on multicast performance, simulation experiments were conducted. In the experiment, 15 scenarios with different MCSs $(0,2,4 \quad \ldots$ 28) were performed and each MCS scenario was repeated 10 times using a set of 10 random seeds. In each scenario, the MCS selected at the beginning of the simulation is kept the same until its end. For more precision and fairness, the same random seed set of values have been used with each MCS experiment repetitions. Moreover, 60 UEs are joining a multicast session and receiving the same video trace file. Other simulation parameters are listed in Table II. To plot the Fairness Index (FI), Packet Loss Ratio (PLR), and the throughput, they must be in the same range. Therefore, throughput and delay were normalized before they were plotted. Whereas, FI and PLR are a ratio, so they were plotted directly without normalization. Furthermore, the FI, PLR, normalized delay, and normalized goodput for each scenario were plotted together as shown in Fig. 2. It seems that the FI remains at maximum value until the selected MCS equals 8, then it starts to drop and continue dropping until it reaches a minimum value when the MCS equals to 28. This is because when the MCS level is less or equal to 8, all UEs can receive the data, but when the MCS becomes larger than 8, few UEs cannot receive the transmitted data, and the number of these UEs increases as the MCS level increases which results in decreasing FI. On the other hand, the goodput is minimum when the MCS equals zero because many packets exceed the maximum allowed delay which causes dropping the packets at the MAC layer. The throughput increases as the MCS level increases until it reaches the maximum throughput value when the MCS equal 12. Then, it starts decreasing because many users cannot decode the data. The PLR is opposite the throughput. However, the throughput reaches the maximum value after the FI starts to decrease and the PLR starts to increase (FI = 0.84, PLR $=0.36$ ).

The delay has inverse relationship with the MCS level. Thus, delay decreases as MCS level increases. Unfortunately, the optimal FI, optimal PLR and optimal throughput are not obtained at the same MCS level. Obviously, the optimal FI occurred when the MCS is less or equal to 8 . The lowest PLR was obtained only when the MCS level equals 8, whereas, the maximum throughput was obtained when the MCS level equals 12. Thus, a trade-off has to be made between throughput, FI, PLR, and delay which depends on the selected MCS. For instance, if the maximum throughput were used, it will select the MCS with level 12; in spite of the degradation of both the FI and PLR.

After plotting the performance of all MCSs in one graph, it is easy to evaluate the performance of any MCS selection mechanism. Thus, a mechanism must conduct with the same scenario, then plotting the obtained results on the same figure (Fig. 2). For instance, to evaluate the performance of existing WCG mechanism, the same scenario was performed using WCG mechanism instead of using a fixed MCS as in previous scenarios. In order to compare the performance of the fixed and WCG, all results were plotted in the same graph. As can be seen from the figure, the WCG mechanism was plotted as star symbol.

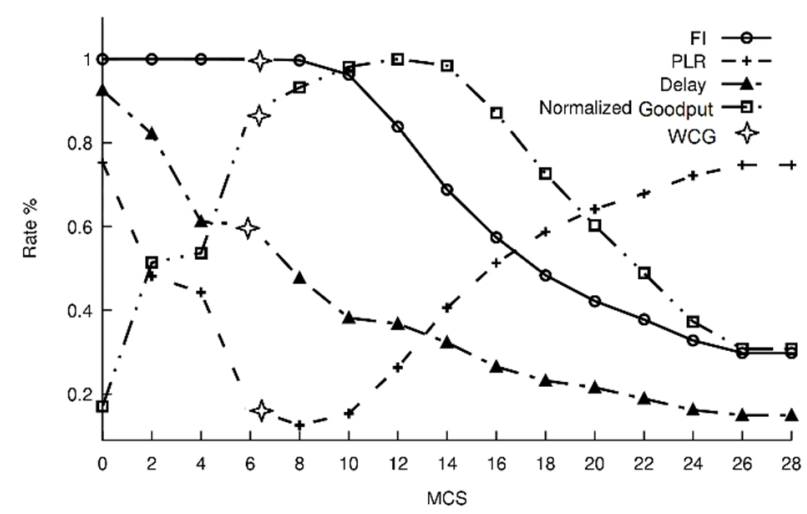

Fig. 2. The performance of eMBMS with WCG scheme at different MCS levels.

\section{B. Average MCS Mechanism}

In this mechanism, users frequently send their CQI feedback to their eNodeB(s) which then maps the CQI to the corresponding MCS level. The eNodeB calculates the Average MCS level using (1) [21]

$$
M C S_{a v g}=\frac{1}{N} \sum_{i=1}^{N} M S C_{i},
$$

where $M C S_{i}$ is the $M C S$ level for user $i$, and $N$ is number of users who successfully sent CQI feedback. The standard deviation of the users' MCS value is defined in (2)

$$
\sigma=\sqrt{\frac{1}{N} \sum_{i=1}^{N}\left(M C S_{i}-M S C_{a v g}\right)^{2}}
$$

This mechanism increases the throughput on account of decreasing FI. However, Average mechanism cannot guarantee the QoS for all users, especially cell edge users. Consequently, those users with low MCS level will suffer high bit error rate.

\section{Median or Standard OMS}

This mechanism calculate the MCS as follows.

Let $\mathrm{MCS}_{1} \leq \mathrm{MCS}_{2} \leq \ldots \leq \mathrm{MCS}_{\mathrm{N}}$ represent the $\mathrm{n}$ descending ordered MCSs of multicast users in group $g$. Let $\alpha=\frac{N}{2}=50 \%$ be the portion of users going to be served.

Thus, the sender will select the rate based on the users with $\alpha$ order 


$$
M C S_{O M S}=M C S_{\alpha} .
$$

\section{Maximum Throughput Mechanism}

In this mechanism, eNodeBselects the MCS level which results in maximum group throughput. To do this, the mechanism will calculate the group throughput depending on each user's MCS. The MCS that results in maximum throughput will be selected without taking into account other users with MCS less than the selected MCS. However, transmitting the data at the rate of the selected MCS will result in high PLR [30]. In spite of the PLR, Maximum Throughput (MT) mechanism does not provide the highest throughput in all cases. For example, if there were free RBs still available after eNodeB transmitted a packet with the rate $r_{i}$, it will be better to utilize them by reducing the selected rate. Hence, more users can receive the data, which, in turn, increases the MG throughput. As a result, MT really provides the maximum throughput, compared to other mechanisms, when network resources are limited [11].

Let $r_{i}$ be the rate associated with $M C S_{i}$. LTE networks offer $15 \mathrm{MCS}$ levels, thus there are 15 different rates can be used to transmit data. Let $A D R_{i}$ be the aggregate data rate of multicast group $g$, which obtained when data transmitted with $M C S_{i}$, and assume there are $N_{i}$ users who can receive the data with $r_{i}$, hence, $A D R_{i}=r_{i} \times N_{i}$. Thus

$$
M C S_{M T}=\arg \max \left\{A D R_{i} \mid 1 \leq i \leq 15\right\} .
$$

\section{The Proposed MCS SElECtion MeChanisM}

The WCG mechanism provides optimal FI between users, however, it degrades the throughput, PLR, and delay. As can be noted from Fig. 2, there is a chance to improve the EMBMS performance by selecting another MCS level bigger than the one selected by WCG. In such case; where few users located at the cell edge while the remaining users located close to the eNodeB, selecting the MCS corresponding to the WCG, as proposed in previous works such as [27], [30], [31], wastes the bandwidth and degrades the E-MBMS performance. In a previous work [20], the authors proposed a mechanism that efficiently selects the MCS level using WCG data based on extreme value theory. The mechanism is proposed to mitigate the limitation of WCG mechanism by using the Standard Deviation (StD) of the users' MCS values to exclude cell edge users (called abnormal users) that are expensive to be covered during the multicast service. However, in this work, instead of excluding those abnormal users, the OMS is combined with their MCS mechanism to serve them. Therefore, the threshold $T_{l o w}$ will be used to split the multicast group into two subsets, normal and abnormal subsets, which are denoted by $M C S_{N G}$, and $M C S_{A G}$ respectively:

$$
\begin{aligned}
M C S_{N G}^{v} & =\left\{M C S_{i} \mid \forall M C S_{i} \geq T_{\text {low }}\right\}, \\
M C S_{A G}^{v} & =\left\{M C S_{i} \mid \forall M C S_{i}<T_{\text {low }}\right\} .
\end{aligned}
$$

Thus, in each transmission, the eNodeB will transmit the data with a rate corresponding to the WCG of the activated subset. eNodeB starts by transmitting the data to the normal subset (using the rate of worst users in this group, which is the lower threshold). then, in the second transmission, eNodeB will transmit the same data to the abnormal group, using the rate of worst users in this group. More transmissions may be needed to make sure that all users receive the data. Steps for the proposed mechanism, called the Modified Worst Channel Group (M-WCG) mechanism are explained in Algorithm 1 below.

Algorithm 1. Modified Worst Channel Group (M-WCG)

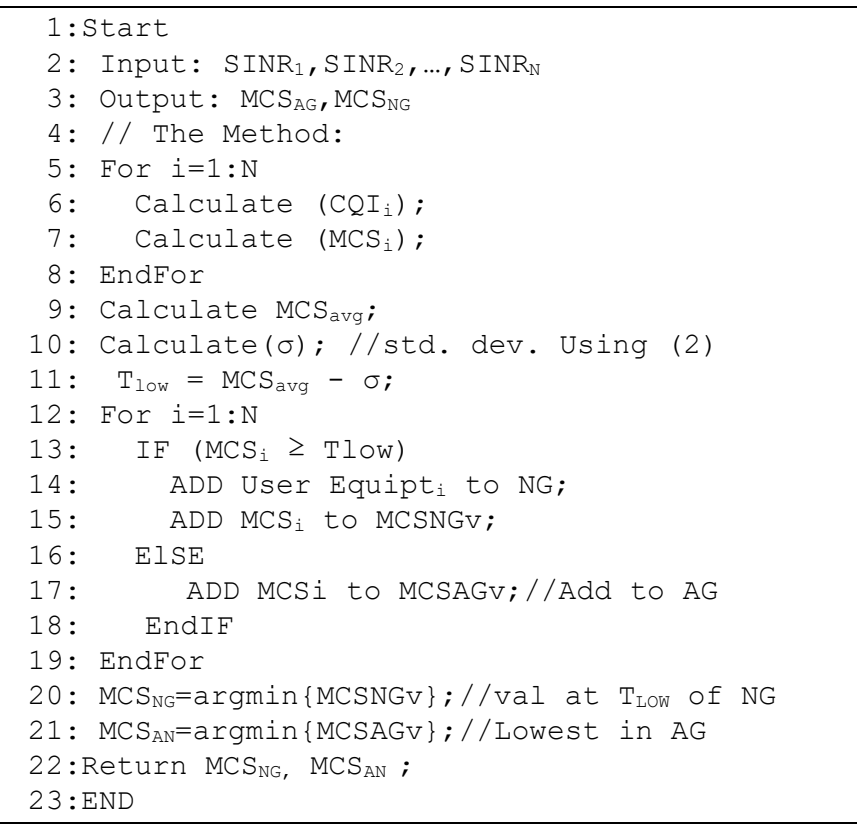

Having done grouping the users into normal and abnormal groups, this work introduces the use of OMS mechanism to provide broadcast services to the users in the abnormal group. This idea improves the fairness level and at the same time maintains the throughput and decreases the Packet Loss Ratio (PLR) as well as the delay. This is attributed to that users with good channel conditions in the normal group will not be affected by the lowest rate of cell edge users

\section{V.EXPERIMENTS SET UP, RESULTS AND DISCUSSION}

\section{A. Experimental Set $U p$}

The experiments are conducted on a computer with the following specifications:

Processor: Genuine Intel(R) CPU U2700@1.30 GHz, single core. RAM: 3 GBytes. Operating System: Fedora 18. Software: LTE simulator (called as LTE-Sim) was used to evaluate the proposed mechanism after extreme modifications and extension for its functions and classes in order to support multicast over LTE network. LTE-Sim is an open source framework simulator developed by Piro and Capozzi [32].

In order to evaluate the performance of the M-WCG mechanism, two simulation experiments were conducted: the first experiment was performed to compare the proposed M-WCG performance with the conventional WCG, MT, Average, Median and D-OMS mechanisms with OMS enabled, and with OMS disabled. In the OMS enabled mode for Average and Medin, the users are also subdivided into normal and abnormal groups using Algorithm 1 above after modifying threshold calculation. Hence, the first transmission is done using the lowest rate determined by the corresponding Average, and Median respectively, while the 
rate of second transmission is determined by the absolute WCG of the abnormal group, like in our proposed M-WCG.

The second experiment was conducted with different max video delay values. Moreover, in each experiment, two different video trace rates are used, low video rate (LVR), and high video rate (HVR), which are indicated as Case 1, and Case 2 respectively. The LVR rate is around $440 \mathrm{kbps}$ whereas the HVR rate is around 3.6 Mbps. In each case, the M-WCG performance was evaluated and the two scenarios; disabled OMS and enabled OMS, were compared, as listed in Table II.

TABLE III. EXPERIMENTS SCENARIOS.

\begin{tabular}{|c|c|c|c|}
\hline Case & Video Rate & Scenario & CMS/OMS \\
\hline Case 1 & LVR & Scenario 1 & CMS \\
\hline & & Scenario 2 & OMS \\
\hline Case 2 & HVR & Scenario 1 & CMS \\
\hline & & Scenario 2 & OMS \\
\hline
\end{tabular}

In the disabled OMS mode, the data is transmitted only one time which is corresponding to the CMS transmission mode. Moreover, the M-WCG performance is compared with other mechanisms, such as the WCG, the Average (AVG), the Median (Standard OMS), MT, and D-OMS, in terms of throughput, BLER, Delay, and FI. The main simulation parameters, as defined in [33], are listed in Table III.

TABLE III. SIMULATION PARAMETERS.

\begin{tabular}{|c|c|}
\hline Parameter & Value \\
\hline Carrier Frequency & $2 \mathrm{GHz}$ \\
\hline Path loss & $\begin{array}{c}\text { PL }(\mathrm{db})=128.1+37.6 \times \log _{10} \mathrm{~d}, \mathrm{~d} \\
\text { in } \mathrm{km}\end{array}$ \\
\hline Thermal noise & $-174 \mathrm{dBm} / \mathrm{Hz}$ \\
\hline Downlink Bandwidth & $3 \mathrm{MHz}$ \\
\hline Symbols for TTI & 12 \\
\hline Sub-Frame Length & $1 \mathrm{~ms}$ \\
\hline Frame Type & FDD \\
\hline eNodeB radius & $1 \mathrm{~km}$ \\
\hline eNodeBPwr Trans. & $43 \mathrm{dBm}$ \\
\hline Modulation Schemes & $\begin{array}{c}\text { QPSK, } 16 \mathrm{QAM}, 64 \mathrm{QAM} \\
(\text { dynamic})\end{array}$ \\
\hline Number of users & $20-100$ \\
\hline Numb. Of E-MBMS group & 1 \\
\hline User transmit. power & $23 \mathrm{dBm}$ \\
\hline User distribution & Random and Uniform \\
\hline User speed & $30 \mathrm{~km} / \mathrm{h}$ \\
\hline User mobility model & Random direction \\
\hline Application flows & Video \\
\hline CQI scheme & $440 \mathrm{kbps}$ \\
\hline
\end{tabular}

\section{B. Experimental Verification}

Since we extended the LTE-Sim with multicast module, it is necessary to verify the simulation model. For this purpose, small experiments are conducted to measure the fitness of the simulation model. According to Sargent [34], three approaches are commonly used to verify the proposed model: Animation; Trace files; and debugging the simulation step by step.

We choose the Trace files approach. The simulation model is verified during the coding stage by verifying the simulation output traces through shell script to check if any of the experiments have been intercepted before the given time. Also we verify through testing the simulation model using a data file with known output. Then we run the simulation model and compare the simulation output with the known output. The verification processes are mainly focused to ensure that the LTE-Sim with E-MBMS functions (such as the scheduling events, random number generator, and random variate generators) together with the conceptual model have been programmed and implemented correctly.

Since the LTE-Sim will be extended to support the EMBMS network, the E-MBMS framework will be verified before the implementation of the proposed mechanisms. The NetBeans (open source software) has been used as an Integrated Development Environment (IDE) for the LTESim to reduce the effect of verification processes, and to make the simulation easier and quicker for extension, debugging, and tracing.

For model fitness testing, we executed five MCS mechanisms (M-WCG, Average, MT, OMS, and D-OMS). For each mechanism we used 5 different delay times and repeated 20 to 100 times. Overall, we conducted 1250 experiment to test the simulation model. We calculate the moving average until achieving $95 \%$ confidence interval. An example of the results is shown in Fig. 3.

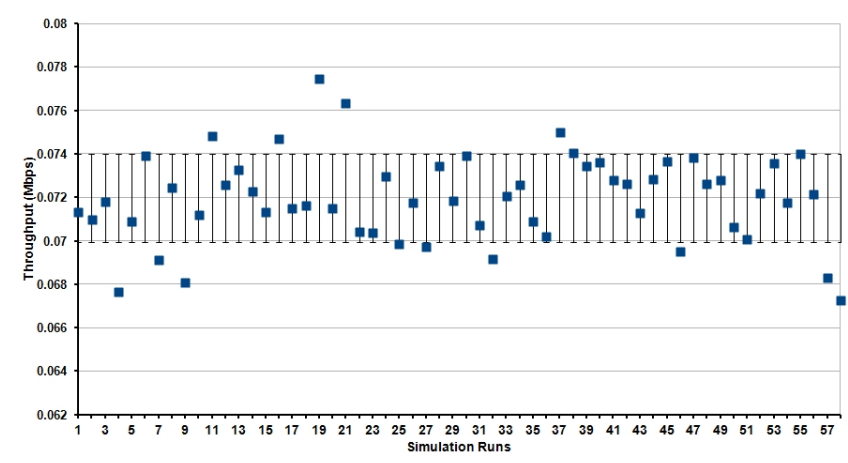

Fig. 3. Example of moving average achieving $95 \%$ confidence interval.

\section{Experiment 1}

In this experiment, a pure multicast and single cell with 100 users involved to evaluate the proposed $\mathrm{M}-\mathrm{WCG}$ performance at two different traffic rates: low rate and high rate.

a) Case 1: Low Video Traffic Rate: Two scenarios with LVR are performed. First scenario was performed to evaluate and compare the M-WCG mechanism performance with enabled OMS scenario, whereas, the second scenario was performed to compare with disabled OMS (known as CMS). The results of both scenarios were plotted together on one graph as shown in Fig. 4(a)-Fig. 4(d). Notice that in these figures, WCG and MT are only tested in CMS (disabled OMS) while D-OMS is only tested in OMS enabled mode.

As shown in Fig. 4(a), the throughput of $\mathrm{M}-\mathrm{WCG}$ mechanism (with disabled OMS) outperforms the throughput of the other mechanisms, even the MT mechanism. The MT mechanism throughput is supposed to be the best among other mechanisms' throughputs. Actually, the MT mechanism performance is degraded because it selects the MCS level that expects to maximize the throughput; therefore, several users fail to decode the received data which causes in high Block Error (BLER) rate 
and leave some free RBs. Indeed, this will not degrade the MT throughput if there is always data needed to be transmitted (e.g. in HVR as in the next case). As a result, it

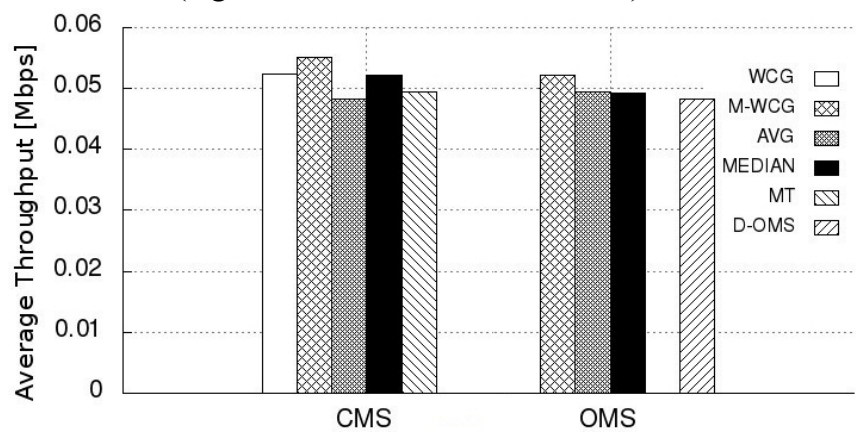

(a)

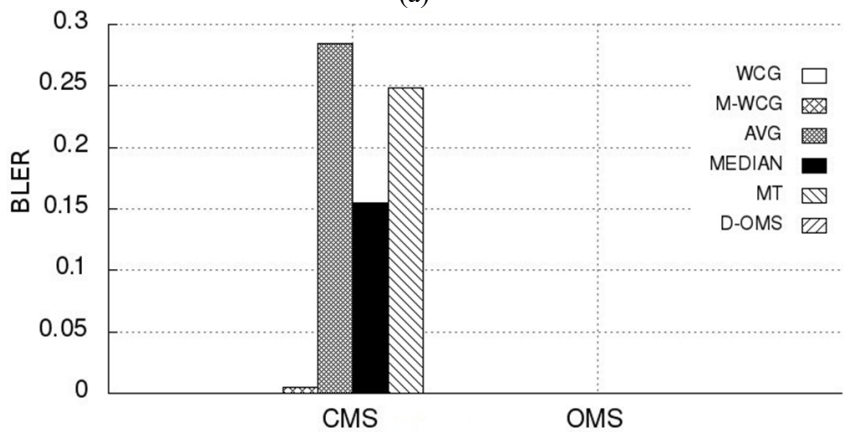

(c) is better to utilize the available RBs as possible by selecting a suitable MCS level which can transmit a packet before it expires.

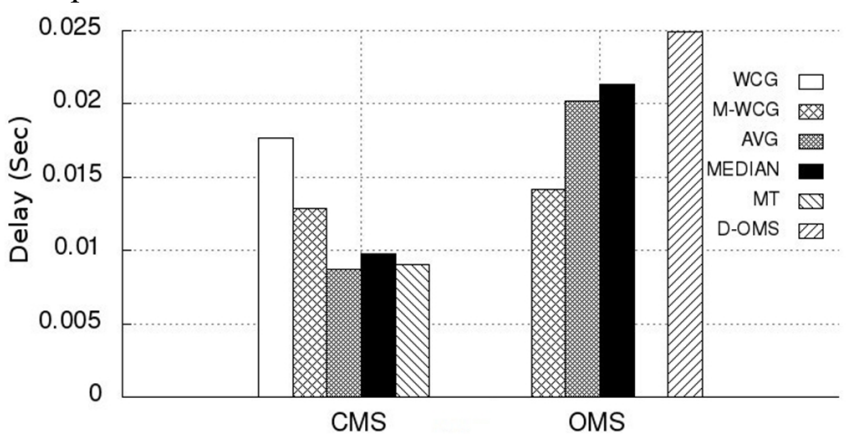

(b)

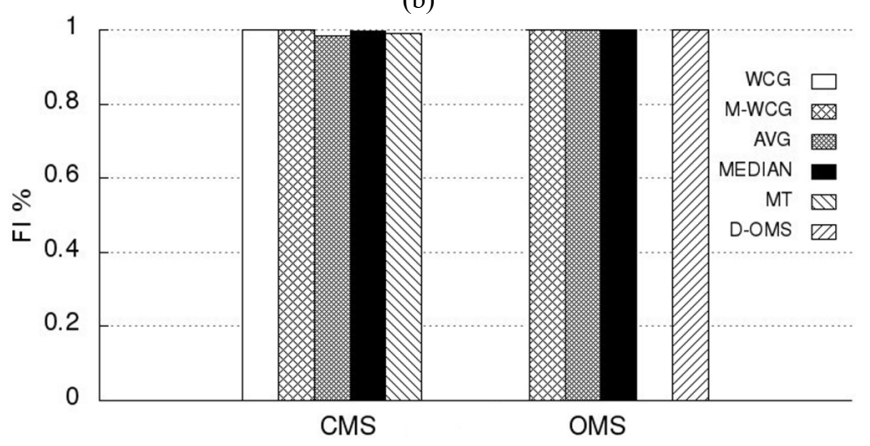

(d)

Fig. 4. Performance comparison of different mechanisms with and without OMS, for LVR: a) throughput; b) delay; c) BLER; d) FI.

Although, WCG mechanism utilizes almost all available $\mathrm{RBs}$, its throughput degrades. The reason behind this is the selected lower MCS level (lower transmission rate) take more time to transmit a packet which causes in long packet queue at the MAC layer. Some packets expires and gets dropped before it is being transmitted. Moreover, the extreme lowest MCS level which is selected by WCG forces other users with high channel gain to scarify there opportunities to receive the data at higher bit rates. This, together with the high packet drop rate at MAC layer causes in WCG performance degradation, especially, in terms of throughput, delay, and PLR. However, the drawback of WCG can be avoided by using M-WCG mechanism which sacrifices those users with extreme lowest MCS levels.

In the second scenario with enabled OMS, as shown in Fig. 4(a) the proposed M-WCG throughput outperforms other mechanisms throughput performance. Actually, the DOMS mechanism performance degrades rather than to be the best because many RBs are used to retransmit the data to users who failed to receive the data in the first transmission. Additionally, the D-OMS mechanism is impractical to be used in real system. The throughput of M-WCG is not much affected by enabled OMS scenario. The difference is around $0.62 \%$. Thus, M-WCG is suitable to be used in both CMS and OMS. However, it is worth to notice that throughputs of all mechanisms with enabled OMS is decreased when comparing with their throughputs with disabled OMS (except the Average mechanism). This is because, in OMS mode, many RBs are used to retransmit the same data to small portion of users who could not receive the data in the first transmission. In contrast, in disabled OMS, these RBs are used to transmit new data which improves the multicast throughput. Figure 4(b) shows the delay of various mechanisms. The highest delay occurs with the D-OMS, whereas the smallest delay occurs with M-WCG. The MWCG provides the lowest delay in OMS case and moderate delay in CMS case. However, the OMS increases the delay in all mechanisms compared to CMS. The reason behind this is that in the OMS case, more time is needed to retransmit the data. The OMS delay increases as the number of retransmission increases.

BLER is one of the important metrics which is used to quantify the performance of MCS selection mechanisms. BLER is a measure of the percentage of the packets which are received with error. As shown in Fig. 4(c) the mechanisms with OMS does not present any BLER. This is because OMS transmits all packets received with BLER several times until all users receive all of the packets. Since, OMS uses the WCG of scheduled user's portion to transmit the packets, users will receive them free of errors. Figure 4(d) shows the Fairness of all methods are close to 1, which means the proposed M-WCG fairness is acceptable.

b) Case 2: High Video Rate: In this case, also two scenarios with HVR were performed. When the HVR is used, free RBs will be limited. First scenario was performed to evaluate and compare the M-WCG performance with enabled OMS, whereas, the second scenario was performed with disabled OMS (known as CMS). The results of both scenarios were plotted together on one graph as shown in Fig. 5(a)-Fig. 5(d).

As shown in Fig. 5(a), in CMS mode, MT, Average, and Median mechanisms outperforms the M-WCG mechanism in term of throughput; on the account of increasing their BLER. In contrary, in OMS mode, the M-WCG mechanism outperforms other mechanisms in terms of throughput, delay, and FI. Figure 5(b) shows the delay of the various mechanisms.

Each mechanism was evaluated with and without 
enabling the OMS. The highest delay occurs with the conventional WCG mechanism. The smallest delay occurs with the Average mechanism in CMS mode, and with MWCG in OMS mode. This is because the Average mechanism transmits the data at high rates, which, in turn, reduces the packet waiting time in the MAC layer queue. Moreover, M-WCG mechanism in OMS mode, provides the minimum delay among the mechanisms. As shown in Fig. 5(c), the highest BLER was obtained from the Average mechanism with value about $30.8 \%$. Also Median and MT provides high BLER with values $16.85 \%, 25.1 \%$ respectively. Nevertheless, these mechanisms provide high MG throughputs as they deliver the data at high rates to a

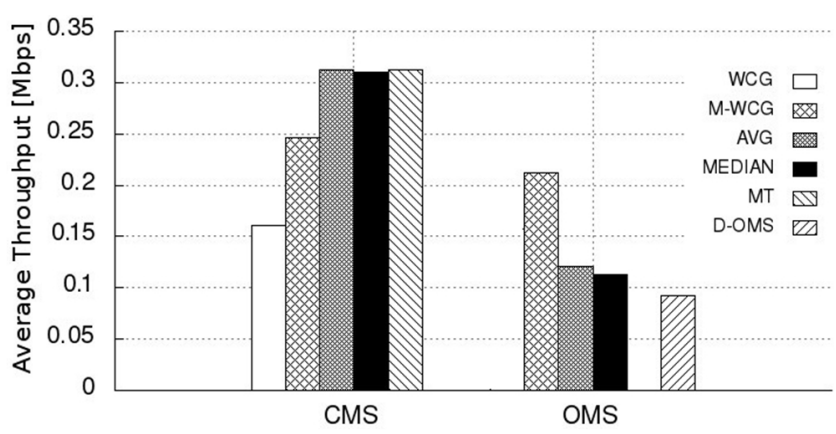

(a)

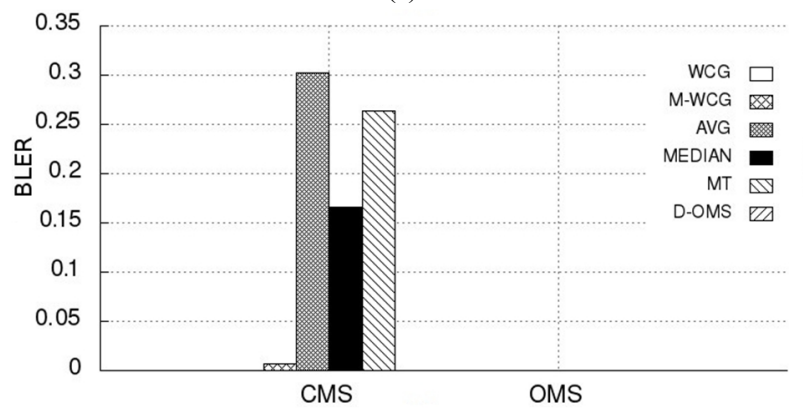

(c)

Fig. 5. Performance comparison of different mechanisms with and without OMS for HVR: a) throughput; b) delay;

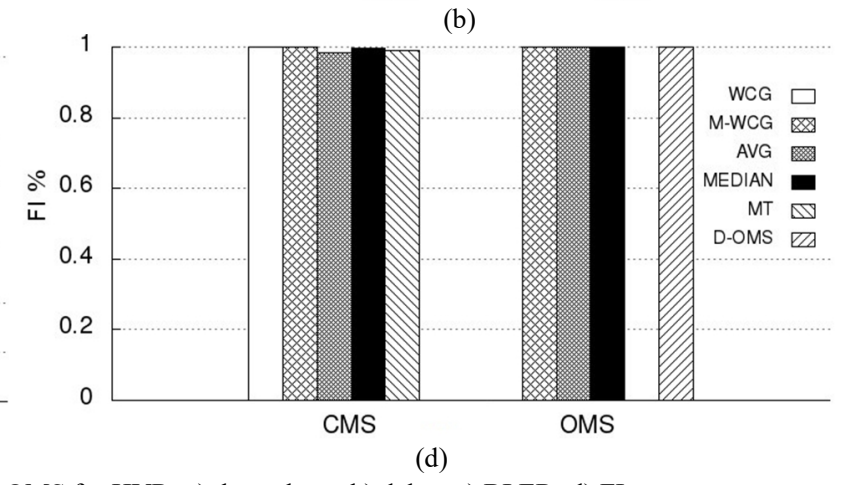

portion of the users who enjoy good channel conditions. On other side, the remaining users who suffer bad channel conditions cannot decode the data reliably, hence, high BLER will occur.

In conclusion, M-WCG outperforms the Median and the Average mechanisms in term of BLER. As shown in Fig. 5(d), all mechanisms with OMS provide optimal FI. This is due to the retransmission procedure which ensures that the transmitted packet is received by all users. Consequently, all multicast users will receive the same data during the session. Hence, all the mechanisms will have a $100 \%$ fairness with OMS, which improves over the situation in their corresponding CMS modes.

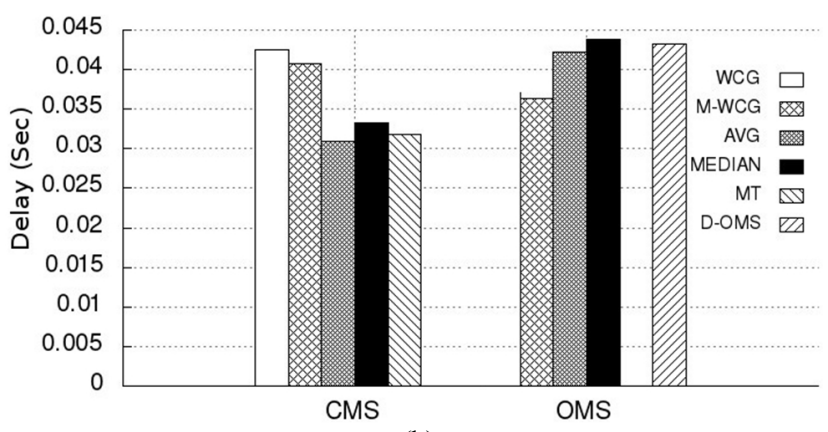

(b)

c) BLER; d) FI.

\section{Experiment 2}

Multimedia applications such as video and VOIP applications are sensitive to packet delay and loss. Thus, transmitted packet must be received before its delay exceeds the preset target delay. If a user receives the packet with delay bigger than the target delay, it will drop the packet. Therefore, to save the bandwidth, eNodeB always checks the packets delay at the MAC queue and drops each packet that exceeds the target delay. Consequently, packet loss is affected by the target delay. Indeed, the packet delay is one of the most scheduling challenging issues especially in real-

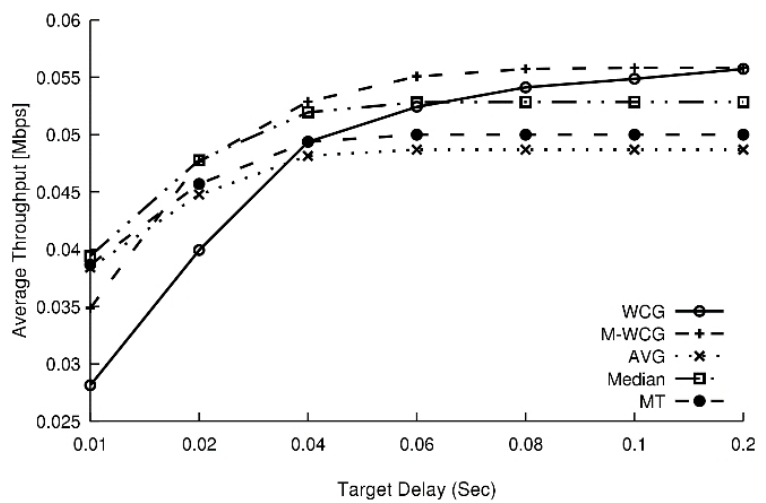

(a) time application. If the scheduler is able to deliver the video packets before they expire, packet loss will be reduced. As a result, high quality video will be reconstructed in real time at the receiver side. As mentioned in [35], for end users quality satisfaction, the video and audio maximum target delay must be less than $300 \mathrm{~ms}$. Furthermore, for interactive video applications the target delay is $200 \mathrm{~ms}$.

In this experiment, the proposed M-WCG is compared with other mechanisms using target video delay in the range of $10 \mathrm{~ms}-200 \mathrm{~ms}$, hence, performance is evaluated at short and long target delay values.

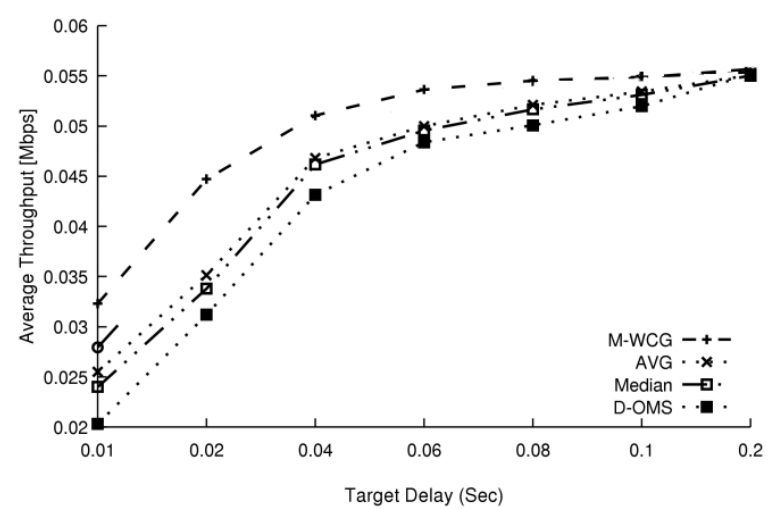

(b)

Fig. 6. Average throughput when low video rate is used in a scenario with: a) disabled OMS; b) enabled OMS 


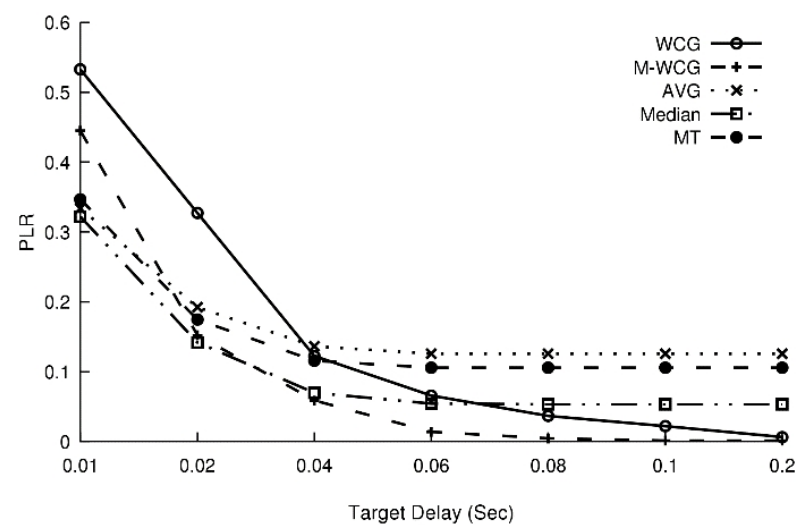

(a)

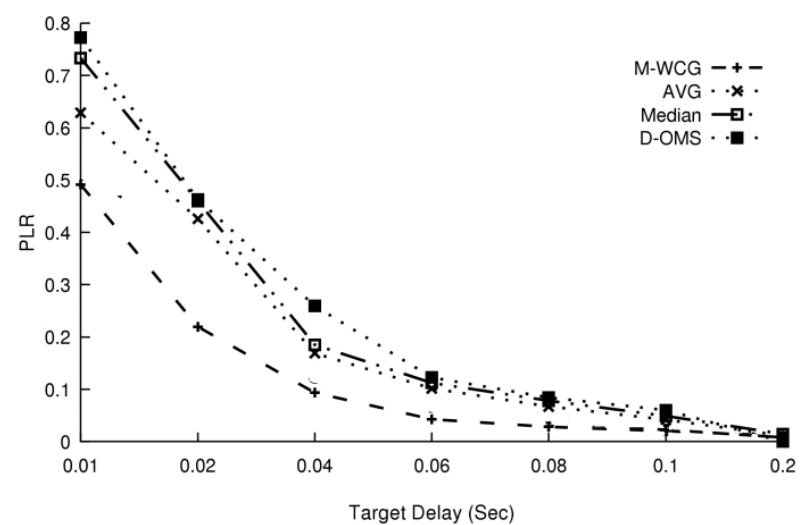

(b)

Fig. 7. PLR when Low Video Rate is used in a scenario with: a) disabled OMS; b) enabled OMS.

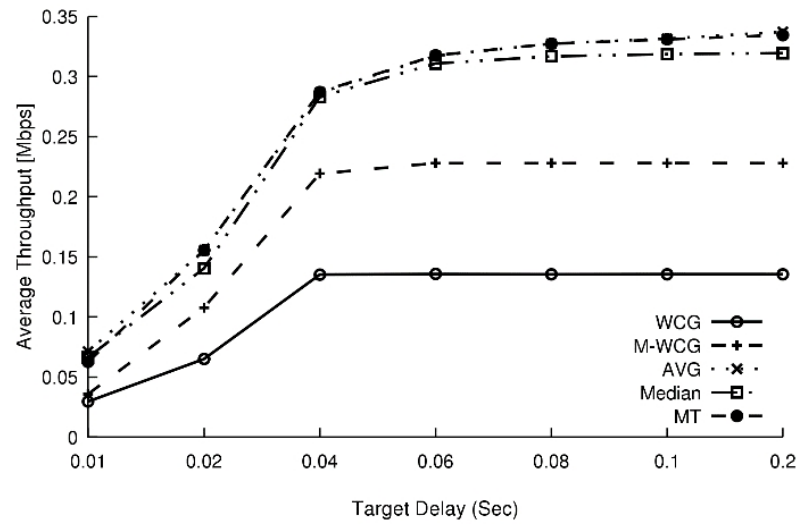

(a)

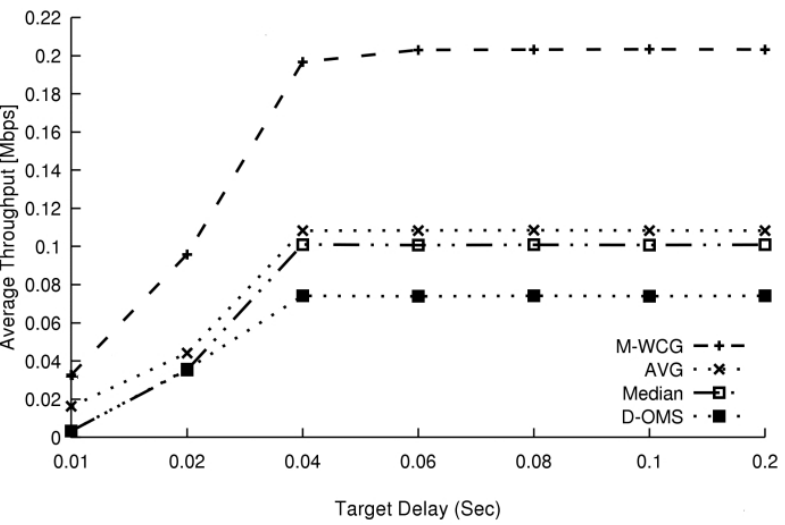

(b)

Fig. 8. Average throughput when High Video rate is used in a scenario with: a) disabled OMS; b) enabled OMS.

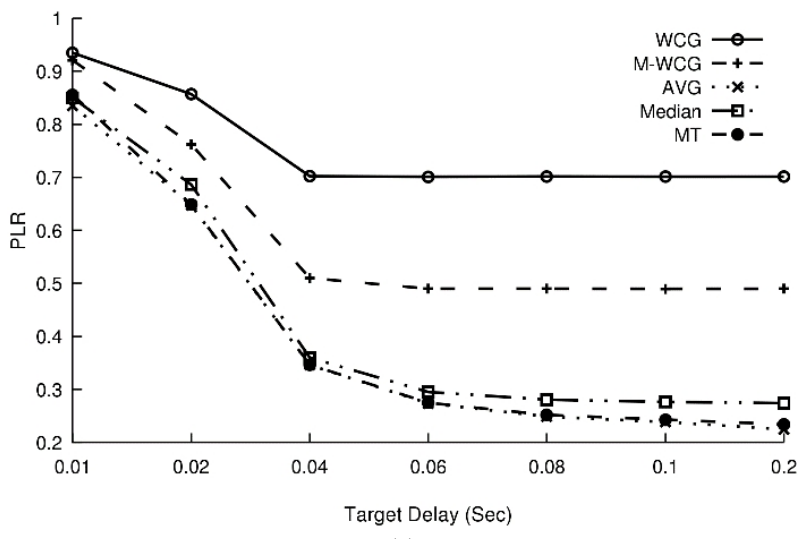

(a)

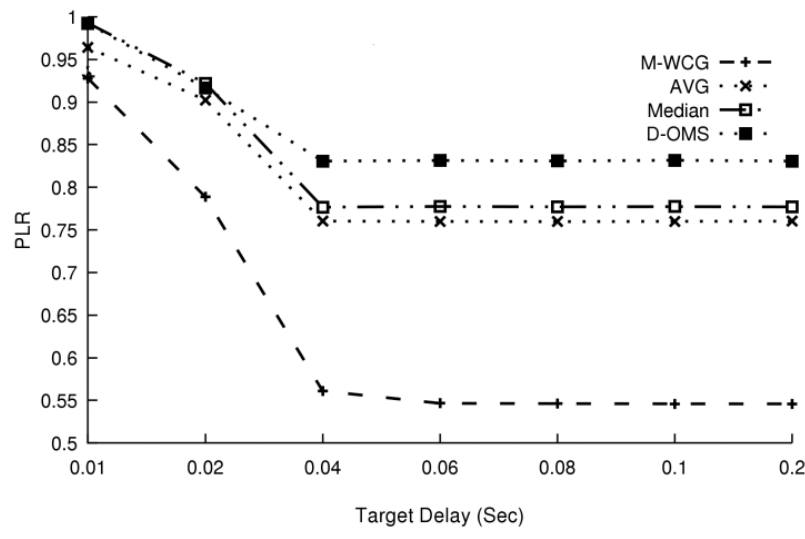

(b)

Fig. 9. PLR with High Video Rate in a scenario with: a) disabled OMS; b) enabled OMS.

The following paragraphs discuss Experiment 2 results:

a) Case 1: Low Video Rate: In this experiment, we compared the proposed mechanism with other mechanisms. Two scenarios were performed with and without enabled OMS. The results of this case are plotted in Fig. 6 and Fig. 7. In general, as shown in Fig. 6, the throughput of all mechanisms increases as the target delay increases either in CMS or in OMS scenarios. In contrary, the PLR decreases as the target delay increases as shown in Fig. 7. However, in CMS scenario, it can be noted that throughput and PLR of Average, Median, and MT did not change after the target delay exceeds 60 milliseconds. This phenomena is because the mechanisms use high transmission rates, which ensures that all packets are transmitted early before they get expired and dropped. On the other side, the WCG and M-WCG still utilize the increase in the target delay to transmit more packets, which in turn, increases the throughput. Finally, at 200 ms delay, the WCG mechanism, with its low transmission rate, have a chance to transmit all the packets on time. As a result, WCG and M-WCG mechanisms provide almost the same throughput and PLR when target delay value approaches the $200 \mathrm{~ms}$ value as shown in Fig. 6-Fig. 7. However, most multimedia applications, such as online video conversation (live streaming), and internet gaming, require lower delay targets. Thus, WCG is not suitable for these applications. In addition, WCG mechanism causes network overhead by splitting each video frame to many small packets each with many headers overhead. In addition, at the receiver side, longer time will be needed to reconstruct the video frame from the received small packets, thus, some packets may expire during the constructing processes. However, in OMS and CMS 
scenarios, it is worth to notice that the proposed mechanism outperforms other mechanisms in terms of throughput and PLR.

b) Case 2: High Video Rate: The simulation parameters and setting are same as in the previous case except that we used here a high video traffic. In this case, with heavy load traffic, most RBs will be assigned for the first transmission, thus, it is difficult to find free $\mathrm{RBs}$ for the OMS retransmission (or, for those mechanisms, which use low rates to send a packet over several TTIs). As can be seen in Fig. 8, the throughputs of all mechanisms (in both cases, with and without enabled OMS) are significantly increased as target delay is increased till the delay reaches $40 \mathrm{~ms}$. However, with target delay larger than $40 \mathrm{~ms}$, throughputs of WCG and M-WCG do not change, whereas, a very small throughput increase occurs with other mechanisms. In fact, the BLER is not affected by changing the target delay, thus, most packets losses happen at the radio link control layer (RLC) and MAC layer (expired packets are dropped before they are scheduled). Consequently, throughput is affected by the packets loss, as can be noted by comparing the throughput and PLR as in Fig. 8 and Fig. 9. Actually, the selected HVR is bigger than to be transmitted over LTE network with $3 \mathrm{MHtz}$ bandwidth even with high coding rate. The arrived packets at the MAC queue are much more than the transmitted packets. That is why all mechanisms provide high PLR. To reduce the PLR, the transmitted video rate should be adapted according to the receiver rate by using cross layer with scalable video coding (SVC) [36] technique, which is not implemented in this paper and is left for future work.

However, in OMS scenario, the proposed M-WCG still outperforms the throughput of the Average and Median mechanisms. In addition, its throughput and PLR follow the D-OMS mechanism, which makes the proposed mechanisms the best to be used in HVR with opportunistic multicast networks.

\section{CONCLUSIONS}

This paper has presented the M-WCG mechanism, which is proposed to enhance multicast video services in LTE and beyond networks. The proposed mechanism was designed to take multicast users dispersion into account to increase the utilization of multiuser diversity and multicast gain. In the M-WCG, a threshold is used to split the multicast users into two subsets according to users' channel conditions. Then, the multiuser diversity is exploited by using the OMS technique. Thus, in each TTI, data is transmitted to the subset with high channel gain. The M-WCG has been evaluated and compared with other existing mechanisms using different video rates and different target delays. The results showed the proposed $\mathrm{M}-\mathrm{WCG}$ outperforms other mechanisms in terms of throughput and delay. For example, the throughput of M-WCG is $6.2 \%$ better than D-OMS with the Median mechanism, and it reduces the delay up to $33.7 \%$ and $17.9 \%$ in low and high video rates respectively. Moreover, in OMS mode, sender continues transmitting the data until all users received it. Therefore, the fairness between all users are almost equal and near to optimal value.

This work contributes towards the improvement of radio resource allocation in multicast, which is important and challenging issues in providing the best multicast service over the LTE wireless networks. As for future work, we will consider the use of the M-WCG with multicast multirate to split users into two or more subgroups with scalable video coding.

\section{REFERENCES}

[1] 3GPP, "Evolved Universal Terrestrial Radio Access (E-UTRA) and Evolved Universal Terrestrial Radio Access Network (E-UTRAN); Overall description; Stage 2," $3^{\text {rd }}$ Generation Partnership Project (3GPP), TS 36.300, Sep. 2013. [Online]. Available: http://www.3gpp.org/ftp/Specs/html-info/36300.htm

[2] 3GPP, "Multimedia Broadcast/Multicast Service (MBMS) Architecture and functional description," $3^{\text {rd }}$ Generation Partnership Project (3GPP), TS 23.246, 2009. [Online]. Available: https://portal.etsi.org/webapp/workprogram/Report_WorkItem.asp?W KI_ID=30531

[3] J. F. Monserrat, J. Calabuig, A. Fernandez-Aguilella, D. GomezBarquero, "Joint delivery of unicast and e-MBMS services in LTE networks", IEEE Trans. Broadcasting, vol. 58, no. 2, pp. 157-167, 2012. DOI: 10.1109/TBC.2012.2191030.

[4] D. Lecompte, F. Gabin, "Evolved multimedia broadcast/multicast service (e-MBMS) in LTE-advanced: overview and rel-11 enhancements", IEEE Communications Magazine, vol. 50, no. 11, pp. 68-74, 2012. DOI: 10.1109/MCOM.2012.6353684.

[5] H. Wang, H.-P. Schwefel, T. S. Toftegaard, "History-based adaptive modulation for a downlink multicast channel in OFDMA systems", in IEEE Wireless Communications and Networking Conf., (WCNC 2008), pp. 1588-1592. DOI: 10.1109/WCNC.2008.284.

[6] H. Won, H. Cai, D. Y. Eun, K. Guo, A. Netravali, I. Rhee, K. Sabnani, "Multicast scheduling in cellular data networks", IEEE Trans. Wireless Communications, vol. 8, no. 9, pp. 4540-4549, 2009. DOI: 10.1109/TWC.2009.080330.

[7] L. Chen, X. Wang, G. He, "Resource allocation with coding schemes for multicast services in single frequency networks", IEEE Int. Conf. Communication (ICC 2012), pp. 5498-5502. DOI 10.1109/ICC.2012.6364666.

[8] L. Zhang, Z. He, K. Niu, B. Zhang, P. Skov, "Optimization of coverage and throughput in single-cell EMBMS", in 70TH IEEE Vehicular Technology Conf. Fall (VTC 2009-Fall), 2009, pp. 1-5. DOI: 10.1109/VETECF.2009.5378877.

[9] P. K. Gopala, H. Gamal, "Opportunistic multicasting", the 38th IEEE Asilomar Conf. in Signals, Systems and Computers, 2004, vol. 1, pp. 845-849. DOI: 10.1109/ACSSC.2004.1399257.

[10] Y.-W. P. H. C.-C. J. K. Tze-Ping Low, Man-On Pun, “Optimized opportunistic multicast scheduling (OMS) over wireless cellular networks", vol. 9, no. 2, pp. 791-801, 2010. DOI: 10.1109/TWC.2010.02.090387.

[11] C. H. Koh, Y. Y. Kim, "A proportional fair scheduling for multicast services in wireless cellular networks", in IEEE 64th Vehicular Technology Conf. (VTC-2006 Fall), 2006, pp. 1-5, DOI: 10.1109/VTCF.2006.225

[12] L. Rong, O. Ben Haddada, S.-E. Elayoubi, "Analytical analysis of the coverage of a MBSFN OFDMA network", in Global Telecommunications Conf. (IEEE GLOBECOM 2008), 2008, pp. 1-5. DOI: 10.1109/GLOCOM.2008.ECP.459.

[13] Y. Sheng, M.-G. Peng, W.-B. Wang, "A novel adaptive modulation and coding strategy based on partial feedback for enhanced MBMS network", The Journal of China Universities of Posts and Telecommunications, vol. 15, no. 1, pp. 48-54, 2008. DOI: 10.1016/S1005-8885(08)60061-8.

[14] G. Araniti, M. Condoluci, A. Molinaro, S. Pizzi, "Radio-aware subgroups formation for multicast traffic delivery in WiMAX networks", IEEE 23rd Int. Symposium on Personal, Indoor and Mobile Radio Communications (PIMRC 2012), Sydney, NSW, Australia, 2012, pp. 477-482. DOI: 10.1109/PIMRC.2012.6362832.

[15] L. Zhang, Y. Wu, W. Li, K. Salehian, A. Florea, G. K. Walker, "Improving LTE eMBMS system spectrum efficiency and service quality using channel bonding, non-orthogonal multiplexing and SFN", IEEE Int. Symposium on Broadband Multimedia Systems and Broadcasting (BMSB 2016), Nara, Japan, 2016, pp. 1-8. DOI: 10.1109/BMSB.2016.7521908.

[16] L. Zhang, Y. Wu, G.K. Walker, W. Li, K. Salehian, A. Florea, "Improving LTE eMBMS with extended OFDM parameters and layered-division-multiplexing", IEEE Trans. Broadcasting, vol. 63 , pp. 32-47, 2017. DOI: 10.1109/TBC.2016.2630269.

[17] A. Awada, E. Lang, O. Renner, K-J. Friederichs, S. Petersen, K. Pfaffinger, B. Lembke, R. Brugger, "Field trial of LTE eMBMS network for TV distribution: Experimental results and analysis", IEEE 
Trans. Broadcasting, vol. 63, pp. 321-337, 2017. DOI: 10.1109/TBC.2016.2630298.

[18] W. Li, Y. Wu, L. Zhang, K. Salehian, S. Lafleche, D. He, Y. Wang, Y. Guan, W. Zhang, J. Montalban, P. Angueira, M. Velez, S-I. Park, J-Y. Lee, H-M. Kim, "Using LDM to achieve seamless local service insertion and local program coverage in SFN environment", IEEE Trans. on Broadcasting, vol. 63, pp. 250-259, 2017. DOI: 10.1109/TBC.2016.2630302.

[19] G. Araniti, M. Condoluci, L. Militano, A. Iera, "Adaptive resource allocation to multicast services in LTE systems", IEEE Trans. on Broadcasting, vol. 59, no. 4, pp. 658-664, 2013. DOI: 10.1109/TBC.2013.2271387.

[20] M. Algharem, M. H. Omar, I. Alghamdi, R. Budiarto, "An efficient modulation and coding scheme selection mechanism for single-cell mode e-MBMS", in Proc. Electrical Engineering Computer Science and Informatics, vol. 2, no. 1, 2015. DOI: 10.11591/eecsi.2.478.

[21] Y. Ji, F. Chen, L. Liu, "MCS selection for performance improvement in downlink TD-LTE system", in Second Int. Conf. Business Computing and Global Informatization (BCGIN 2012), Shanghai, China, 2012, pp. 687-690. DOI: 10.1109/BCGIN.2012.184.

[22] J. Fan, Q. Yin, G. Li, B. Peng, X. Zhu, "MCS selection for throughput improvement in downlink LTE systems", in Proc. 20th Int. Conf. in Computer Communications and Networks (ICCCN 2011), Maui, HI, USA, 2011, pp. 1-5. DOI: 10.1109/ICCCN.2011.6005743.

[23] X. Chen, H. Yi, H. Luo, H. Yu, H. Wang, "A novel CQI calculation scheme in LTELTE-A systems", Int. Conf. in Wireles. Communications and Signal Processing (WCSP 2011), Nanjing, China, 2011, pp. 1-5. DOI: 10.1109/WCSP.2011.6096767.

[24] 3GPP, "Evolved Universal Terrestrial Radio Access (E-UTRA); Physical layer procedures," 3rd Generation Partnership Project (3GPP), TS 36.213, Oct. 2013. [Online]. Available: http://www.3gpp.org/ftp/Specs/html-info/36213.htm

[25] R. Fantacci, D. Marabissi, D. Tarchi, I. Habib, "Adaptive modulation and coding techniques for ofdma systems", IEEE Trans. Wireless Communications, vol. 8, no. 9, pp. 4876-4883, 2009. DOI: 10.1109/TWC.2009.090253

[26] R. Cohen, G. Grebla, L. Katzir, "Cross-layer hybrid FEC/ARQ reliable multicast with adaptive modulation and coding in broadband wireless networks", IEEE/ACM Trans. Networking (TON 2010), vol. 18, no. 6, pp. 1908-1920, 2010. DOI: 10.1109/TNET.2010.2050902.
[27] J. Kim, D.-H. Cho, "Enhanced adaptive modulation and coding schemes based on multiple channel reporting for wireless multicast systems", in IEEE 62nd Vehicular Technology Conf., (VTC-2005Fall), Dallas, TX, USA, USA, vol. 2, 2005, pp. 725-729. DOI: 10.1109/VETECF.2005.1558019.

[28] Y.-C. Liang, C.-C. Chou, H.-Y. Wei, "Modeling and analysis of applying adaptive modulation coding in wireless multicast and broadcast systems," Wireless Networks, vol. 17, no. 5, pp. 1373-1386, 2011. DOI: 10.1109/VETECS.2010.5493660.

[29] G. T. R. WG2, "MBMS Modulation and Coding State Selection", 3rd Generation Partnership Project (3GPP), TS R2-061985, Jun. 2006.

[30] S. Bochrini, C. Bouras, V. Kokkinos, "Efficient MCS selection mechanisms for multicasting over LTE networks", in 6th Joint IFIP Wireless and Mobile Networking Conference (WMNC 2013), Dubai, United Arab Emirates, 2013, pp. 1-8. DOI: 10.1109/WMNC.2013.6548994

[31] A. Alexiou, C. Bouras, V. Kokkinos, A. Papazois, G. Tsichritzis, "Spectral efficiency performance of MBSFN-enabled LTE networks", in IEEE 6th Int. Conf. Wireless and Mobile Computing, Networking and Communications (WiMob 2010), Niagara Falls, ON, Canada, 2010. pp. 361-367. DOI: 10.1109/WIMOB.2010.5645042.

[32] G. Piro, L. A. Grieco, G. Boggia, F. Capozzi, P. Camarda, "Simulating LTE cellular systems: an open-source framework", IEEE Trans. Vehicular Technology, vol. 60, no. 2, pp. 498-513, 2011. DOI: 10.1109/TVT.2010.2091660

[33] 3GPP, "Physical layer aspect for evolved universal terrestrial radio access (UTRA)," 3rd Generation Partnership Project (3GPP), TR 25.814, Oct. 2006. [Online]. Available: http://www.3gpp.org/ftp/Specs/html-info/25814.htm

[34] R. Sargent, "Verification and validation of simulation models", in Proc. Simulation Conference (WSC 2011), Austin, TX, USA, USA, 2011, pp. 183-198. DOI: 10.1109/WSC.2009.5429327.

[35] 3GPP, "Quality of service, charging and policy control (Release 11)", 3rd Generation Partnership Project (3GPP), TS 23.203, Nov. 2012, [Online] Available: http://www.etsi.org/deliver/etsi_ts/123200_123299/123203/11.07.00_ 60/ts_123203v110700p.pdf

[36] H. Schwarz, D. Marpe, T. Wiegand, "Overview of the scalable video coding extension of the H.264/AVC standard", IEEE Trans. Circuits Syst. Video Technol., vol. 17, no. 9, pp. 1103-1120, 2007. DOI: 10.1109/TCSVT.2007.905532. 